\title{
Educación Patrimonial y su inclusión en la Formación Inicial Docente: una tarea en desarrollo*
}

\author{
Heritage Education and its inclusion in Initial Teacher Training: A \\ work in progress
}

\author{
${ }^{1}$ Enrique Muñoz Reyes \& ${ }^{2}$ Lorena P. López Torres
}

Muñoz, E., López, L. (2021). Educación Patrimonial y su inclusión en la Formación Inicial Docente: una tarea en desarrollo. Revista Convergencia Educativa, (10-extra), diciembre, 7-23. https://doi.org/10.29035/rce.s10.7

[Recibido: 02 marzo, 2021 / Aceptado: 07 julio, 2021]

\begin{abstract}
RESUMEN
En este artículo proponemos una reflexión general sobre la pertinencia de la Educación Patrimonial en la Formación Inicial Docente, para propender a la elaboración de una actividad curricular que entregue a los docentes en formación, elementos conceptuales y procedimentales sobre patrimonio. Esperamos que su inclusión asegure una educación integral a quienes, como ciudadanos, podrán preservar y valorar esta herencia, y enseñar a sus futuros alumnos a apreciarla, en tanto experiencia comunitaria y social. Con esta finalidad, buscamos proponer posibles formas de abordarla a partir de la revisión de la literatura sobre educación patrimonial e, inicialmente, del análisis del perfil de egreso de las carreras de Pedagogía en Educación Básica con Mención y de Pedagogía en Lengua Castellana y Comunicación de la Universidad Católica del Maule. Pretendemos levantar elementos preliminares que orienten la Formación Inicial Docente de la UCM hacia la incorporación de una perspectiva de enseñanza sobre el territorio que incorpore principios de formación ciudadana y una mirada en torno al patrimonio natural, material y cultural inmaterial local y regional que genere el desarrollo de aprendizajes contextualizados y significativos en los alumnos y las alumnas.
\end{abstract}

Palabras clave: Patrimonio, educación patrimonial, formación inicial docente, educación ciudadana.

* Parte de este artículo fue presentado como ponencia en las I Jornadas Internacionales de Investigación en Didáctica y Formación Inicial Docente UCM 2020.

1 Centro de Investigación en Educación para la Justicia Social, Facultad de Ciencias de la Educación, Universidad Católica del Maule. Curicó, Chile. https://orcid.org/0000-0001-6880-4716 | emunoz@ucm.cl

2 Centro de Investigación en Educación para la Justicia Social, Facultad de Ciencias de la Educación, Universidad Católica del Maule. Talca, Chile, http://orcid.org/0000-0003-2769-5001 | lplopez@ucm.cl 
Muñoz, E., López, L. (2021). Educación Patrimonial y su inclusión en la Formación Inicial Docente: Una tarea en desarrollo. Revista Convergencia Educativa, (10-extra), diciembre, 7-23. https://doi.org/10.29035/rce.s10.7

\begin{abstract}
In this article we propose a general reflection on the relevance of Heritage Education in Initial Teacher Training, in order to promote the development of a curricular activity that provides teachers in training with conceptual and procedural elements on heritage. We hope that its inclusion ensures a comprehensive education of teachers in training, who, as citizens, will be able to preserve and value this heritage, and teach their future students to appreciate it, as a community and social experience. To this end, we seek to propose possible ways of approaching it from the review of the literature on heritage education and, initially, from the analysis of the graduation profile of the Pedagogía en Educación Básica con Mención and Pedagogía en Langua Castellana y Comunicación of the Universidad Católica del Maule. We intend to raise preliminary elements that guide the Initial Teacher Training of the UCM towards the incorporation of a teaching perspective on the territory that incorporates principles of citizen training and a look around the local and regional natural, material and cultural heritage that generates development. contextualized and meaningful learning in male and female students.
\end{abstract}

Key words: heritage, heritage education, initial teacher training; citizen education.

\title{
INTRODUCCIÓN
}

Al considerar las transformaciones sociales, tecnológicas, culturales, políticas y económicas de la sociedad actual, la formación inicial docente en la tercera década del siglo XXI enfrenta diversos y complejos desafíos. En este contexto, la educación patrimonial, con sus objetos de estudio, materiales e inmateriales, en tanto bienes culturales, debe ser considerada en la enseñanza de los niños niñas, y jóvenes de las escuelas, colegios y universidades de Chile, en función de desarrollar en ellos su sentido de pertenencia e identidad. Esta es una idea primigenia e innovadora que enriquece la Formación Inicial Docente (FID) como también la formación profesional en otras áreas o disciplinas del conocimiento.

El proyecto del que deriva este artículo es pionero en nuestro país en el ámbito de la educación superior, dado que hasta el momento toda concepción o transposición pedagógica que se ha hecho sobre educación patrimonial, ha sido desarrollada en el ámbito informal en los establecimientos educacionales, por ejemplo, en talleres, charlas, exposiciones, entre otras actividades. Esta propuesta, en cambio, se orienta a formalizar su enseñanza a partir de su incorporación como materia regular, reflexiva y crítica en la transversalidad de la formación de los estudiantes de pedagogía. Pretendemos que las profesoras y profesores en formación adquieran herramientas didácticas en torno a la educación patrimonial para ser incluidas en sus prácticas profesionales y en su futuro desempeño como docentes.

En este contexto, la Ley N²0.903 que crea el Sistema de Desarrollo Profesional Docente, y que entró en vigencia en 2016, introdujo gradualmente requisitos más exigentes para ingresar a las carreras de pedagogía, aumentando el desafío para las universidades que forman profesores, promoviendo el desarrollo continuo, creando el acompañamiento inicial e introduciendo un sistema de evaluación que comienza en forma previa al ejercicio profesional. Los estándares FID -actualmente en revisión- permiten también ver 
Muñoz, E., López, L. (2021). Educación Patrimonial y su inclusión en la Formación Inicial Docente: Una tarea en desarrollo. Revista Convergencia Educativa, (10-extra), diciembre, 7-23. https://doi.org/10.29035/rce.s10.7

el panorama transversal de las carreras de pedagogía y cómo aportan al debate actual y coyuntural en el que nos encontramos. En este sentido rescatamos el estándar FID N 4 "Genera un ambiente inclusivo para el aprendizaje" (s.p.), en el que están incluidos elementos relacionados con cultura, historia e identidad como se señala a continuación:

Identifica y analiza distintas formas de trabajo que favorecen relaciones de integración, inclusión y colaboración, considerando la diversidad de las y los estudiantes, expresada en etnia, género, religión, características socioculturales, entre otras. Comprende la importancia de un ambiente inclusivo para el aprendizaje, demostrando respeto y valoración por las historias, culturas, identidades y lenguajes de niños, niñas, jóvenes, adultos y sus familias (s.p.).

Por su parte, la Ley $\mathrm{N}^{\circ} 20.911$ integra y complementa las definiciones curriculares nacionales en esta materia, brindando a los estudiantes la preparación necesaria para asumir una vida responsable en una sociedad libre y de orientación hacia el mejoramiento integral de la persona humana, como fundamento del sistema democrático, la justicia social y el progreso. El objetivo del plan de formación ciudadana es: "Fomentar en los estudiantes la valoración de la diversidad social y cultural del país" (p. 1).

Los diferentes espacios garantizados por la educación formal para la formación de los estudiantes delimitan la situación en la que se encuentra la enseñanza sobre patrimonio, siendo insuficientes las acciones emprendidas para contribuir globalmente a su valoración, tanto en la formación del alumnado como en la formación del profesorado, razón por la cual, la inclusión y trascendencia de la Educación Patrimonial (EP) se presenta como un desafío valorable para el sistema educativo, pues contribuye al desarrollo integral de las personas en sus comunidades.

\section{DEFINICIÓN DE PATRIMONIO CULTURAL}

Las principales definiciones de patrimonio las recoge y entrega la Organización de las Naciones Unidas (ONU) a través de su organismo para la Educación, la Ciencia y la Cultura (UNESCO) e identifica el patrimonio mundial como:

el conjunto de bienes culturales y naturales que hemos heredado de nuestros antepasados y que nos permiten entender y conocer la historia, las costumbres y las formas de vida hasta el momento actual. [...] es la base sobre la cual la humanidad construye su memoria colectiva y su identidad, es lo que nos hace identificarnos con una cultura, con una lengua, con una forma de vivir concreta (UNESCO, 2004, p. 3).

Actualmente se entiende como patrimonio cultural aquel que involucra el patrimonio material, natural e inmaterial, pues constituye "el legado que recibimos del pasado, que vivimos en el presente y que transmitiremos a las generaciones futuras" (UNESCO, 2004, p. 3). El agregado "cultural" responde a su vínculo con la sociedad que brinda sentido al patrimonio al reconocerlo y valorarlo; las obras o bienes 
Muñoz, E., López, L. (2021). Educación Patrimonial y su inclusión en la Formación Inicial Docente: Una tarea en desarrollo. Revista Convergencia Educativa, (10-extra), diciembre, 7-23. https://doi.org/10.29035/rce.s10.7

patrimoniales se aproximan a la ciudadanía y le dan un sentido de identidad colectiva. Como concepto vivo y en constante cambio, comprende como centro de sus preocupaciones las obras materiales, no materiales y tradicionales, así como también a sus cultores, es decir, al patrimonio vivo, perseverando en su protección y existencia, ya que una vez desaparecidos no pueden ser recuperados; a estos bienes se le confiere "protección internacional" (UNESCO, 1954, p. 8) para prevenir el menoscabo que significaría su desaparición para toda la humanidad.

Además, la UNESCO propone diferentes normativas como convenciones, recomendaciones y declaraciones para concretar objetivos comunes y jurídicos entre las naciones que se adhieren para su aplicación a nivel nacional e internacional otorgando apoyos, métodos y normas. Las más importantes son la Convención sobre la Protección del Patrimonio Mundial, Cultural y Natural (1972) y la Convención para la Salvaguarda del Patrimonio Cultural Inmaterial (2003), ${ }^{1}$ Convención sobre la Protección y Promoción de la Diversidad de las Expresiones Culturales (2005). Estas además se reconocen por la mayoría de los países del orbe y se consideran parte sustancial del trato, cuidado y difusión de los bienes patrimoniales, entiéndase edificaciones, lugares, objetos y personas de cada nación tanto dentro de sus fronteras como hacia el exterior.

En el contexto de este artículo, sumamos la definición que entrega el Servicio Nacional de Patrimonio Cultural dependiente del Ministerio de las Culturas, las Artes y el Patrimonio:

El patrimonio cultural es un conjunto determinado de bienes tangibles, intangibles y naturales que forman parte de prácticas sociales, a los que se les atribuyen valores a ser transmitidos, y luego resignificados, de una época a otra, o de una generación a las siguientes. Así, un objeto se transforma en patrimonio o bien cultural, o deja de serlo, mediante un proceso y/o cuando alguien -individuo o colectividad-, afirma su nueva condición (DIBAM, 2005, s. p.).

En este sentido, por tanto, el patrimonio cultural no constituye algo definitivo y estático, al contrario, es el resultado de un proceso complejo y dinámico, sus significados y sentidos van cambiando, al relacionarse con una cultura que los contextualiza, los recrea e interpreta. En este contexto, las personas al conocer y transformar la realidad, permiten el surgimiento de nuevas interpretaciones y nuevos usos patrimoniales. Si en un momento de la historia nacional el concepto de patrimonio estuvo referido sólo a los monumentos materiales, ahora considera nuevas tipologías tales como, patrimonio intangible, etnográfico o industrial. Además, se ha atribuido mayor atención a las artes de la representación, las lenguas

1 Esta Convención ha sido suscrita por alrededor de 150 Estados y se complementa con otros instrumentos internacionales relacionados con el patrimonio cultural como la Declaración de los Derechos Humanos (1948); el Convenio OIT 169 sobre Pueblos Indígenas y Tribales (1989); la Declaración de las Naciones Unidas sobre los Derechos de los Pueblos Indígenas (2007) y la Agenda 2030 para el Desarrollo Sostenible (2015). Sobre esta última nos referiremos más adelante. 
Muñoz, E., López, L. (2021). Educación Patrimonial y su inclusión en la Formación Inicial Docente: Una tarea en desarrollo. Revista Convergencia Educativa, (10-extra), diciembre, 7-23. https://doi.org/10.29035/rce.s10.7

y la música tradicional, como también a los sistemas filosóficos, espirituales y de información que constituyen el marco de dichas creaciones.

\section{¿QUÉ ENTENDEMOS POR EDUCACIÓN PATRIMONIAL?}

La noción de Educación Patrimonial -postulada en primera instancia por Olaia Fontal (2003) -, implica un proceso sistemático de trabajo y colaboración al poner en primer lugar el conocimiento de los sujetos participantes por sobre el imperio del objeto patrimonial entendido como único bastión de prestigio cultural. En forma complementaria con esta idea se puede rescatar la siguiente conceptualización acerca de Educación Patrimonial:

[...] un proceso pedagógico centrado en las percepciones, conocimientos y valores que subyacen en una sociedad. De esta manera, el bien patrimonial se convierte en un recurso para el aprendizaje, capaz de conectar al ciudadano con su diversidad cultural y su entorno social. Para ello, es necesario trabajar con los valores culturales locales más próximos a las personas, para luego proyectar los valores universales. El diseño, planificación y ejecución de acciones educativas tendrían como objeto de estudio el diseño de estrategias y recursos educativos centrados en las personas y no en el bien cultural (García, 2009, p. 274).

En consecuencia, el fomento de los procesos educativos relacionados con el patrimonio a través de metodologías que involucren la participación de la comunidad, que logren incluir su visión, experiencias y contenidos, que aseguren la transmisión de conocimiento conceptual, así como también, el fomento de habilidades y competencias de la ciudadanía, posibilitará que se desarrollen procesos de enseñanza orientados a lograr aprendizajes significativos y colaborativos. Desde esta perspectiva, la educación se comprende como un desafío necesario y constante que implica una estructuración continua de la persona humana, de su conocimiento y sus aptitudes, junto con el desarrollo de su capacidad reflexiva y su acción transformadora. Debe permitirle tomar conciencia de sí misma y de su medio ambiente, invitándola a desempeñar su función social en su comunidad a través de su trabajo (Delors, 1996, p. 15).

En esta oportunidad nos situamos ante el patrimonio cultural como una creación humana que consiste en:

[...] la herencia de símbolos que los hombres se han dado a lo largo de los tiempos y en diferentes espacios geográficos. Esas creaciones con formas constituidas de materialidades diversas, así como las expresiones del arte, las obras del pensamiento y la ciencia; sea de la industria y las tecnologías. Todas las expresiones de los pueblos son manifestaciones del espíritu creador del hombre, que se consignan en cosas signos, algunas de las cuales, las más significativas para la comunidad, derivan en símbolos. El hombre es espíritu encarnado y por ello, lo que procede de él, tiene materialidad, por más que ésta, conformada, sea indicadora y seña de una comprensión 
Muñoz, E., López, L. (2021). Educación Patrimonial y su inclusión en la Formación Inicial Docente: Una tarea en desarrollo. Revista Convergencia Educativa, (10-extra), diciembre, 7-23. https://doi.org/10.29035/rce.s10.7

mayor del sentir intelectivo y aún de las más altas abstracciones del pensar y del espíritu (Hernández, 2020, p. 79).

Para Ibarra y Ramírez (2014) los aportes de la educación patrimonial a la sociedad, a la ciudadanía y al sistema educativo permiten, en primer lugar, que la comunidad proteja su legado histórico-cultural con ayuda de expertos; en segundo lugar, que pueda entenderse este en términos de las competencias que promueve porque fortalece el sentido crítico, la reflexión y la formación ciudadana; y, por último, que refuerza el desarrollo cultural local a través de la promoción de procesos de construcción de identidad territorial. En esta elaboración es necesario destacar que la memoria tiene una función relevante, puesto que:

[...] la memoria común hace presente lo custodiado, rememorándolo una y otra vez. ¿Qué hace presente? Hace presente o actual, la herencia y memoria humana. La actualización del pasado en el presente puede darse por la virtualidad del recuerdo que la memoria consigue recrear. El espíritu humano supera y trasciende los tiempos. Pero esa superación del pasado se lleva a cabo, porque la presencia del pretérito es relevante y actual (Hernández, 2020, p. 79).

Ahora bien, ¿qué se entiende por memoria? Es una respuesta que implica complejidad. Existen reflexiones que abordan esta situación desde una perspectiva analítica y comprensiva, señalando que

La memoria no es una reliquia de museo, silenciosa, una mera huella del pasado, custodiada por celosos curadores e historiadores. La memoria es una entidad hipercompleja, desde un punto de vista epistemológico, y carga con una abultada polisemia [...] Vive en nuestra psiquis individual y colectiva; se encuentra en las arenas de lo cotidiano y también en la vida social, política, económica y cultural. [...] Afín con el cuerpo que le hemos dado, la memoria se concibe como columna vertebradora de la construcción de nuestra identidad, personal y colectiva. Los temas de memoria, por ende, son aquellos que nos movilizan como sociedad, en especial a la hora de mirar comprensiva y críticamente el pasado, a la luz de un presente y de los requerimientos de construcción para un proyecto futuro, dando sentido a la existencia y a nuestras experiencias (Ayala en Ramis \& Peña, 2019, pp. 102-103).

\section{EDUCACIÓN PATRIMONIAL EN LA FORMACIÓN INICIAL DOCENTE (FID)}

Creemos que la Educación Patrimonial es una posibilidad de exploración de su entorno y de su identidad para los niños y jóvenes estudiantes de todos los niveles educativos. La labor de los profesores y de los docentes en formación será clave para guiarlos en el conocimiento y cuidado del patrimonio local, regional y nacional. Con ese norte, la educación patrimonial tiene como misión que las personas se identifiquen con la identidad nacional; inculca valores importantes como el respeto, la empatía y la comprensión, y amplía, considerablemente, los conocimientos propios del cuidado de los bienes culturales que las comunidades 
Muñoz, E., López, L. (2021). Educación Patrimonial y su inclusión en la Formación Inicial Docente: Una tarea en desarrollo. Revista Convergencia Educativa, (10-extra), diciembre, 7-23. https://doi.org/10.29035/rce.s10.7

deben preservar. La educación patrimonial recurre a la diversidad cultural y al entorno social de los estudiantes, para que ellos puedan participar en la construcción de su propia memoria patrimonial. De esta forma, su incorporación, con la guía de los profesores, ampliará el capital cultural de los estudiantes y, en consecuencia, contribuirá a su formación integral.

La preocupación puesta en los bienes patrimoniales desde la FID puede significar un motor de cambio del currículum nacional, que incentive el tratamiento de lo patrimonial, ya no de forma aislada y solo restringida a espacios informales fuera del espacio escolar. Su llegada a las aulas de clases ha de impactar en la sociedad dado que el trabajo realizado en los establecimientos educativos irradiará a la comunidad circundante por medio de la transmisión de aprendizajes que recojan las experiencias, memorias y herencias de esa colectividad.

Uno de los aportes de la Educación Patrimonial, tanto para profesores como para estudiantes y padres de las comunidades educativas es, desde una dimensión afectiva y simbólica, fomentar y garantizar "el conocimiento, preservación y transmisión del patrimonio en todas sus dimensiones" (Ibáñez et al., 2015, p.12).

Las mallas de las carreras de pedagogía pueden ofrecer espacios que deben ser utilizados en pos de la inclusión formal de la Educación Patrimonial en la FID. Esto puede concretarse a través de la incorporación de algunos de sus elementos basales en algunas actividades curriculares específicas o de la creación de nuevas actividades curriculares, considerando las adecuaciones o los rediseños curriculares que las carreras planteen cuando sea necesario. En el mismo sentido, el eje de prácticas progresivas podría beneficiar al otorgar a los docentes en formación alternativas de tratamiento de materias patrimoniales en los recintos educativos en los que intervengan haciendo uso de las herramientas de la educación patrimonial. Por último, la educación continua o el postgrado puede ofrecer diplomados y programas de magíster que se aboquen al campo de investigación y acción enfocado en la problemática patrimonial. Este es un ámbito multidisciplinar que en forma paulatina está siendo robustecido y que plantea un atractivo particular para investigadores, profesionales, profesores y estudiantes de diferentes vertientes, pues cruza miradas transdisciplinarias para la búsqueda y resolución de problemáticas concernientes.

\section{EL PATRIMONIO EN EL MODELO FORMATIVO DE LA UNIVERSIDAD CATÓLICA DEL MAULE}

La pertinencia de nuestra reflexión preliminar está en sintonía con los principios y orientaciones del Modelo Formativo de la Universidad Católica del Maule (UCM, 2014), que presentan como propósito esencial la entrega de una educación con un sello distintivo, innovador y transformador del entorno sociocultural. A través de las competencias profesionales adquiridas, el egresado será capaz de rescatar la identidad de las comunidades locales y regionales con su patrimonio; con ello, desarrollará su sentido de pertenencia social e histórica-cultural hacia la región. 
Muñoz, E., López, L. (2021). Educación Patrimonial y su inclusión en la Formación Inicial Docente: Una tarea en desarrollo. Revista Convergencia Educativa, (10-extra), diciembre, 7-23. https://doi.org/10.29035/rce.s10.7

El Modelo Formativo de la UCM apunta al desarrollo de la identidad regional, por tanto, es pertinente y coherente elaborar e implementar un módulo de educación patrimonial, el cual contribuirá con la formación profesional de los docentes en formación. Además, se fomentará y desarrollará el sentido de pertenencia local y regional de los estudiantes, los profesores y los apoderados de las comunidades, junto con establecer una vinculación bidireccional con el medio. De acuerdo al Proyecto Educativo Institucional (2014), la UCM se define como "una comunidad académica consagrada a la búsqueda de la verdad, y al cultivo de la dignidad humana y del patrimonio cultural, por medio de la docencia, la investigación y la extensión" (p. 24). En consonancia con esta declaración es relevante destacar lo que se menciona en dicho documento en la dimensión Pedagógico-Educativa:

Concebimos la educación como un proceso de formación integral, mediante la articulación sistemática y crítica de la cultura, la que entendemos como un rico patrimonio por asimilar, así como un elemento vital y dinámico del cual la educación forma parte. De este modo, la cultura se hace educativa. Una educación que no cumpla esa función, limitándose a elaboraciones prefabricadas, se convertirá en un obstáculo para el desarrollo de la personalidad de los estudiantes (p. 24).

En consideración de lo señalado en el proyecto educativo institucional creemos que las proyecciones que estamos formulando en este artículo adquieren mayor relevancia y sentido, puesto que nos señalan que las proyecciones de crear una actividad curricular sobre Educación Patrimonial para la FID encuentran su fundamento en la misión institucional, aun cuando falta una operacionalización de estas intenciones. En el mediano plazo pretendemos abordar la elaboración de este curso para responder a los requerimientos de la UCM en cuanto a la vinculación con los territorios y las identidades del Maule.

\section{ALGUNAS EXPERIENCIAS DE EDUCACIÓN PATRIMONIAL EN LA FID DE LA UCM}

En los últimos años -2015 a la fecha-, hemos impulsado iniciativas que aportan de forma sustancial a incorporar la Educación Patrimonial en la Formación Inicial Docente. Estas instancias han decantado en ejercicios de promoción de aprendizajes más colaborativos, democráticos y transdisciplinarios, con las que hemos buscado generar un impacto en la formación de profesores. El objetivo último ha sido que los jóvenes profesionales lleven consigo las herramientas, los conocimientos y las reflexiones surgidas y las proyecten a su vida laboral para activar un aprecio positivo sobre el patrimonio en las aulas chilenas. La Educación Patrimonial permite que cultivemos experiencias en los profesores que enriquezcan su visión sobre el escenario educativo local y regional con un sentido de unidad y coherencia que les permita articular conocimientos, estrategias y habilidades para su provecho en las comunidades educativas con las que estén en contacto.

Entre las iniciativas a las que nos referimos se encuentra el Seminario de Historia Local, Memoria y Patrimonio realizado por primera vez en 2016 y que a lo largo de los años se ha dedicado a promover la 
Muñoz, E., López, L. (2021). Educación Patrimonial y su inclusión en la Formación Inicial Docente: Una tarea en desarrollo. Revista Convergencia Educativa, (10-extra), diciembre, 7-23. https://doi.org/10.29035/rce.s10.7

historia local, la memoria y el patrimonio de la provincia de Curicó y de la región del Maule. A través de la presencia de especialistas en la materia se comparte sobre temáticas de interés e investigaciones con estudiantes, profesores, investigadores y público en general. En 2017 se crea el Grupo de Investigación Poliana. Escuela \& Imaginarios -integrado por estudiantes y egresados de la carrera de Lengua Castellana y Comunicación- que ha levantado inicialmente la creación del Archivo Histórico de la Escuela San Miguel de Talca en estrecha colaboración con la comunidad educativa; incluyó la realización de talleres de educación patrimonial para alumnos de $7^{\circ}$ básico con quienes se reconoció el patrimonio educativo y familiar. La instalación de placas informativas de las escuelas normales de Chile, en 2017, dio paso a la creación de la Sala Museo Profesoras y Profesores Normalistas de Chile, espacio museístico que se levantó en colaboración con los docentes normalistas de Curicó en el 2018 para recuperar su memoria histórica, pedagógica y social a sus profesores y que permite a los visitantes reconocer el legado que dejaron en las escuelas y colegios de los campos, pueblos y ciudades a lo largo del país.

Las iniciativas aquí presentadas marcan hitos en nuestra trayectoria que han permitido abrir paso a la Educación Patrimonial en la FID, pues retratan de manera abreviada las repercusiones de un trabajo realizado que ha sido paulatino y sistemático. Por un lado, rescatamos el patrimonio escolar y, por otro, la memoria pedagógica y sociocultural de la región a partir de la investigación realizada y de la construcción de propuestas.

A estas acciones de relevamiento patrimonial, debemos sumar los artículos publicados, libros, capítulos de libros y tesis de licenciatura ${ }^{2}$ dirigida que nos han permitido profundizar en nuestras inquietudes; estos aportes se han creado en conjunto con los profesores en formación de ambas carreras de pedagogía señaladas. Precisamente la tesis de grado titulada Lineamientos sobre Educación Patrimonial en la Formación Inicial Docente: Perspectiva de profesores, egresados y estudiantes de Pedagogía en Lengua Castellana y Comunicación (2019) de las ex estudiantes de Pedagogía en Lengua Castellana y Comunicación, Ignacia Castro y Priscila López, contribuyó de forma sustancial a la visibilización de nuestros intereses por consolidar un espacio para la Educación Patrimonial. En su investigación recogieron información directa de

2 Podemos mencionar algunos artículos: “Archivo emocional y corpus fotobiográfico. La construcción de la memoria visual de una comunidad escolar" (2019); "Las relaciones pasado-presente en la enseñanza de la historia: en busca del sentido de los aprendizajes históricos (2017); el libro Archivo Histórico de la Escuela San Miguel. Rescate del patrimonio educacional de la Escuela San Miguel, Talca (2019), y el capítulo de libro "Aquí se viene a estudiar, de aquí se sale a servir: Escuela Normal Cecilio Imable Yens de Curicó: periodo1948-1973" (2021). Además, las tesis de licenciatura: El imaginario de la educación normalista en Chile (1928-1974) como referente para la actual Formación Inicial Docente (FID) (2019) y Alcances de la educación patrimonial chilena en el marco del desarrollo de las competencias transversales en estudiantes de séptimo y octavo año básico (2018). Por último, los Seminarios de Título: El cementerio municipal de la comuna de Curicó como recurso pedagógico para la enseñanza de la historia local (2015) e Historia de la Plaza de Armas de Curicó (2015). 
Muñoz, E., López, L. (2021). Educación Patrimonial y su inclusión en la Formación Inicial Docente: Una tarea en desarrollo. Revista Convergencia Educativa, (10-extra), diciembre, 7-23. https://doi.org/10.29035/rce.s10.7

actores -académicos y estudiantes de la carrera- que dan cuenta de sus consideraciones sobre patrimonio, y de sus opiniones y comentarios sobre la incorporación de la Educación Patrimonial en la formación del profesorado de la Institución. Sin duda, se trata de una investigación que, por su alcance, significa un avance preliminar en lo que respecta a los intereses de este artículo.

En esta investigación, las autoras revisaron el perfil de egreso y las competencias generales y específicas del programa para indagar la relación de la Formación Inicial Docente y la Educación Patrimonial, sumando a su análisis la observación de los Estándares Orientadores de la Formación Inicial Docente, los Planes y Programas y otros documentos ministeriales para sumarlos a su reflexión. De ahí surge una mirada crítica a los escasos alcances curriculares del Ministerio de Educación y de la legislación nacional respecto de la Educación Patrimonial.

Algunos de los resultados de la información recogida, puntualizan en la importancia de referenciar algunos elementos constituyentes de la formación docente de la UCM. El más mencionado por los entrevistados guarda relación con el sello identitario regional que mencionan por estimar que se orienta hacia lo patrimonial; lo entienden como una competencia general de la Institución que debiese ser cultivada con mayor dedicación, de forma de asegurar su incorporación explícita como objetivo y competencia en las planificaciones y syllabus de la carrera de Pedagogía en Lengua Castellana y Comunicación. Prestan igual atención a que las otras pedagogías de la casa de estudios incorporen postulados de la Educación Patrimonial. Esta atención podría incidir en las matrices curriculares de las carreras de pedagogía y, por ende, en sus perfiles de egreso. En este orden, el proyecto formativo de la carrera de Pedagogía Básica con Mención, hace explícita la referencia a la identidad sociocultural regional, la que se puede vincular con un componente patrimonial a trabajar durante el itinerario formativo: "calidad integral, identidad sociocultural regional, responsabilidad social e investigación en los itinerarios formativos que articulan pre y postgrado" (2016, s. p.).

Otra evidencia es el trabajo realizado en algunos seminarios de título realizados por ex estudiantes de la carrera de Pedagogía en Educación Básica con mención en Ciencias Sociales de la UCM sede Curicó. Por ejemplo, el seminario "Historia de la Plaza de Armas de Curicó", realizado por las ex alumnas Hellen Cabrera, Bárbara Muñoz y Paula Reyes (2015), quienes indagaron acerca de qué sabían los estudiantes de quinto, sexto y séptimo básico de dos centros educativos acerca de la historia y el patrimonio del espacio público céntrico de la ciudad. Como resultado de la investigación, la mayoría de los estudiantes consultados desconocía el origen histórico de los elementos patrimoniales de la Plaza, debido a que el currículum de historia, geografía y ciencias sociales de educación básica no contempla objetivos de aprendizaje que aborden la realidad del entorno local y regional. Al finalizar este seminario se propone realizar una visita guiada por la Plaza de Armas con la finalidad de que los estudiantes identifiquen sus elementos característicos y logren comprender el origen histórico de estos y su valor patrimonial. 
Muñoz, E., López, L. (2021). Educación Patrimonial y su inclusión en la Formación Inicial Docente: Una tarea en desarrollo. Revista Convergencia Educativa, (10-extra), diciembre, 7-23. https://doi.org/10.29035/rce.s10.7

De esta forma, las pedagogías vinculadas con la identidad cultural de la región, expanden las posibilidades de interacción de los docentes en formación con bienes medulares de la zona como su literatura, artesanía, tesoros vivos y su memoria educacional, entre otros; riquezas abiertas al estudio y recuperación desde el ámbito pedagógico. Para ello, el docente debe entender su labor como un compromiso con su comunidad en tanto profesional y ciudadano, por lo que debe "tener las competencias para desarrollar la educación sobre el patrimonio, tanto en el aula como fuera de esta, y de manera transversal, independiente de las distintas áreas disciplinares. [...] el desafío es que todos los docentes puedan vincularse con las resignificaciones patrimoniales del país, cuestión que se encuentra en constante dinamismo a raíz de la contingencia social" (Castro \& López, 2020, pp. 108-109). Lo puntualizado en esta última investigación, grafica el camino recorrido por las intervenciones e investigaciones previas realizadas y que continúan expresándose, no solamente en este artículo, sino también en las proyecciones que formulamos en esta oportunidad, de forma parcial.

La misión de incorporar la Educación Patrimonial en la Formación Inicial Docente significa, a nuestro parecer, que los futuros profesores se presenten ante sus alumnos con un rol distintivo y modélico y que comparta con ellos su preocupación y aprecio por el patrimonio. De esta manera, el profesor revelará a sus alumnos:

su propia imagen a imitar de un ciudadano preocupado y responsable, consciente de su entorno y de sus necesidades, interesado en el rescate de la historia local y personal de ellos y de sus familias, de sus bienes patrimoniales y de la importancia de su conservación y cuidado. Lo mismo debemos hacer los académicos que junto a ellos nos sumamos a esta significativa tarea (López et al., 2021, p. 19).

La propia experiencia en torno al patrimonio que adquieran los profesores en formación, estimulada en su paso por la universidad, les infundirá la confianza suficiente para apropiarse de los bienes patrimoniales y a acercar a otros a su conocimiento y puesta en valor. En sus entornos educativos laborales les permitirá identificar problemas y levantar propuestas en beneficio del patrimonio local y regional que beneficie a las comunidades.

\section{Propuesta inicial}

En este siglo XXI, existe un espacio formativo a desarrollar en la FID en una doble dimensión, la formación ciudadana y la educación patrimonial. Esto en consonancia con la nueva Ley $\mathrm{N}^{\circ} 20.911$ de Formación Ciudadana, promulgada en el año 2016 que establece como una de sus finalidades "[...] Contribuir hacia la formación de ciudadanos, con valores y conocimientos que fomenten el desarrollo del país, con una visión del mundo centrada en el ser humano, como parte de un entorno natural y social". Por tanto, se deduce que es necesario que el estudiante conozca, analice y valore el medio en que vive, para lo cual requiere que sus profesores estén formados en esta perspectiva. 
Muñoz, E., López, L. (2021). Educación Patrimonial y su inclusión en la Formación Inicial Docente: Una tarea en desarrollo. Revista Convergencia Educativa, (10-extra), diciembre, 7-23. https://doi.org/10.29035/rce.s10.7

El aporte que hace la Educación Patrimonial es introducir en la formación de profesores y en consecuencia en la enseñanza y el aprendizaje de niños, de jóvenes y adultos, el patrimonio natural, material e inmaterial como objeto de interés con la finalidad de conocerlo, valorarlo y difundirlo. Esto supone la participación activa de los estudiantes, sus padres y otros actores de las comunidades en que ellos viven, porque al ocuparnos de los bienes patrimoniales los debemos hacer desde nuestra responsabilidad como ciudadanos, porque todos somos colaboradores de su preservación, puesta en valor y difusión. Este es el rol que como ciudadanos y ciudadanas debemos cumplir ante los bienes patrimoniales.

Ahora bien, ¿quién define qué es patrimonio? Las propias comunidades deben determinar en forma participativa lo que consideran bienes patrimoniales, para ser percibidos, asumidos y valorados como propios, como elementos que los identifican y otorgan sentido de pertenencia.

Existen propuestas teóricas que plantean criterios orientadores de la educación ciudadana para ampliar el marco curricular sobre la formación en ciudadanía, entre ellos el siguiente: "Recuperar las memorias populares y objetos patrimoniales en todos los ámbitos posibles" (Pagés en Ramis, 2019, pág.12), es decir, la centralidad de la memoria y lo patrimonial en la enseñanza y el aprendizaje de la ciudadanía adquiere relieve en el siglo XXI, lo que abre una posibilidad teórica y práctica de educar a niños, jóvenes y adultos en una dimensión dual: patrimonial y ciudadana. Existe un soporte en la legislación que establece la obligatoriedad de implementar un plan de formación ciudadana en las escuelas y colegios de Chile, lo que redunda en la necesidad de un soporte nuevo relacionado con la Educación Patrimonial, por lo que proponemos incluirla en forma explícita, intencionada, coherente y sistemática en la FID.

¿Qué aportará la educación patrimonial en el sistema educativo chileno? Creemos que favorecerá el desarrollo de un pensamiento crítico y propositivo en niños y jóvenes respecto de su realidad social, cultural, económica y política en los territorios que habitan. Además, al explorar, reconocer, poner en valor y difundir los bienes patrimoniales materiales e inmateriales de sus comunidades, se convertirán en protagonistas de sus procesos de aprendizaje. Para esto, es necesario que el aula se convierta en un espacio democratizador desde el que se promuevan y practiquen la inclusión, tolerancia y aceptación. Así los estudiantes podrán comprender cómo están conformados desde el punto de vista identitario, con lo cual desarrollarán su sentido de pertenencia.

Del presente artículo surge la inquietud consecuente de realizar una investigación exploratoria de tipo cualitativo (Hernández et al., 2010) relacionada con las concepciones acerca de patrimonio y Educación Patrimonial por parte de estudiantes de Pedagogía en Educación Básica con Mención y Pedagogía en Lengua Castellana y Comunicación. En una primera etapa, se recogerá información de los estudiantes de pedagogía acerca de las concepciones de patrimonio, educación patrimonial e identidad para establecer el estado de la cuestión y se contrastará con lo que el currículum de las carreras de Pedagogía en Educación Básica con Mención y Pedagogía en Lengua Castellana y Comunicación, ofrece a los estudiantes en relación con estas materias. A partir de los datos recogidos, en una segunda etapa, buscaremos elaborar una propuesta de 
Muñoz, E., López, L. (2021). Educación Patrimonial y su inclusión en la Formación Inicial Docente: Una tarea en desarrollo. Revista Convergencia Educativa, (10-extra), diciembre, 7-23. https://doi.org/10.29035/rce.s10.7

formación en educación patrimonial para las carreras de pedagogía. Esta propuesta se elaborará igualmente considerando las aportaciones teóricas de expertos en la materia, lo que permitirá consolidarla.

Consideramos que para poner en valor los bienes patrimoniales, hacerlos circular y ser reconocidos, debe elaborarse un plan formativo que brinde herramientas para la comprensión y difusión del patrimonio, para ser desarrollado en escenarios tanto formales (escuelas y universidades) como informales (centros comunitarios o juntas de vecinos). De acuerdo a esto, distinguimos la relevancia de la educación patrimonial para la formación integral de los estudiantes, como actores sociales conscientes de lo que significa el patrimonio en conjunto con el conocimiento, las competencias y los valores necesarios para la conservación de estos bienes. En este aspecto, la educación patrimonial postula una enseñanza integral que busca fortalecer el desarrollo de la identidad cultural al interior de la comunidad educativa y al mismo tiempo reconocer y valorar la identidad de la realidad que rodea al establecimiento o comunidad educativa; así mismo, se aboca a estimular el pensamiento crítico y consciente en los estudiantes -y también en los docentes y en los padres y apoderados- sobre el pasado, el presente y el futuro de su escuela y su comunidad. En consecuencia, una persona que logre tomar conciencia de sí misma para desempeñar una función social, reconocerá en la educación patrimonial el vínculo que contribuye a la cohesión ciudadana para la construcción de su memoria histórica. Esta perspectiva de educación patrimonial ha de colaborar en la forma en que convivimos como sociedad, como parte de una voluntad de vivir juntos reconociendo y respetando nuestra diversidad cultural.

El interés por el progreso de la educación hacia una experiencia social compuesta a partir de conocimientos teóricos, prácticos y procedimentales, confirmará la necesidad de ver a los docentes en formación, a los estudiantes y profesores del sistema escolar, como personas que aprenden continuamente y que reflexionan sobre la realidad en una comunidad específica. Tanto la investigación como la praxis en torno a la educación patrimonial, permitirán generar propuestas pedagógicas en el área de la Formación Inicial Docente (FID) de la Universidad Católica del Maule. Esta reflexión en torno al patrimonio, con sus componentes disciplinarios, didácticos y pedagógicos, incorporará conocimientos todavía no sistematizados en el currículum formal de las pedagogías, que creemos, formará parte de estas a través de la creación de una actividad curricular, de la realización de investigaciones de síntesis de grado, de la conformación de grupos de investigación entre académicos y profesores en formación, y de la vinculación con el medio a través del eje de prácticas con los establecimientos educacionales de la región.

\section{CONCLUSIONES}

Las reflexiones planteadas en este artículo están alineadas con el modelo formativo de la UCM, el cual, entre sus finalidades, se orienta al desarrollo de la identidad regional, por tanto, la creación e implementación de un curso de educación patrimonial será pertinente. Junto a esto, se contribuirá con el sentido de pertenencia de los estudiantes, profesores y apoderados de las comunidades de la región, y al mismo tiempo, se podrá 
Muñoz, E., López, L. (2021). Educación Patrimonial y su inclusión en la Formación Inicial Docente: Una tarea en desarrollo. Revista Convergencia Educativa, (10-extra), diciembre, 7-23. https://doi.org/10.29035/rce.s10.7

establecer una vinculación bidireccional con el medio. Estamos rescatando referencias teóricas para sustentar nuestra propuesta, las que nos permitirán elaborar los fundamentos conceptuales para la elaboración de la actividad curricular acerca de patrimonio. Junto a esto, incorporaremos los elementos metodológicos a partir de algunas de las experiencias de investigación e intervención patrimonial que hemos realizado, por ejemplo, la Sala-Museo de las profesoras y profesores normalistas en la sede Curicó de la UCM y el Archivo Escolar de la Escuela San Miguel de Talca.

Las investigaciones revisadas se orientan en recomendar la inclusión de lo patrimonial en la educación formal, principalmente en la educación básica y media. Esto nos hace pensar que, para su adecuado traslado a las salas de clases, debe iniciarse un proceso de sensibilización en los docentes en formación, quienes, de forma transversal y transdisciplinaria, armados con competencias y herramientas didácticas y pedagógicas, dentro del marco de lo patrimonial, podrán brindar la experiencia de enseñanza y aprendizaje a sus alumnos en los establecimientos educacionales.

La investigación acerca de la educación patrimonial en la formación inicial docente en el contexto del siglo XXI, aportará elementos para la elaboración e implementación de un módulo en las carreras de pedagogía. Así se contribuirá con la formación integral de los docentes en formación, enriqueciendo su quehacer en su futuro rol como profesores y profesoras en ejercicio. Además, a través de la educación patrimonial, se colaborará con respuestas a las demandas de reconocimiento social de los diferentes actores y grupos que reflejan la diversidad cultural de nuestro territorio nacional. El paso siguiente será establecer una tipología de concepciones acerca de la educación patrimonial que manejan los estudiantes de pedagogía, integrando el material teórico suficiente para plasmar los idearios sobre patrimonio y su enseñanza en la educación superior. Con esto se pretende diferenciar los niveles de importancia que se atribuyen a los objetos patrimoniales, a su conservación, difusión y a su enseñanza a terceros, es decir, a los estudiantes en el sistema escolar, y las personas en general. Mediante el análisis de los datos se buscará crear un modelo de educación patrimonial que sea incorporado en la formación de profesores de la UCM a través de actividades curriculares transversales.

Consideramos necesaria y pertinente la educación patrimonial en la formación inicial docente, la que contribuirá a la educación integral de los docentes en formación, y fomentará el desarrollo de un trabajo colaborativo con profesores en ejercicio. Creemos que la educación patrimonial debe ser incluida en actividades educativas, partiendo por la FID, que privilegie el contexto social y cultural, local y regional. Debe ser entendida como un proceso de aprendizaje que promueva el conocimiento, el pensamiento crítico y la formación ciudadana, puesto que poner en valor, rescata y preserva el patrimonio de todos.

La mirada germinal que ofrecemos en este artículo, gira en torno a los reconocimientos iniciales que los estudiantes de Pedagogía en Lengua Castellana y Comunicación de la Universidad Católica del Maule (UCM) y Pedagogía en Educación General Básica con Mención tienen respecto a la Educación Patrimonial, en correspondencia con sus conocimientos y habilidades parciales sobre esta. Si perseveramos en el 
Muñoz, E., López, L. (2021). Educación Patrimonial y su inclusión en la Formación Inicial Docente: Una tarea en desarrollo. Revista Convergencia Educativa, (10-extra), diciembre, 7-23. https://doi.org/10.29035/rce.s10.7

tratamiento de lo patrimonial en todas sus dimensiones desde la FID, los docentes en formación podrán generar vínculos con sus alumnos, colegas, apoderados y otros actores sociales de establecimientos de práctica o laborales a futuro, para estimularlos el descubrimiento de los bienes patrimoniales conservados o por rescatar desde la articulación de todos los sentires e intereses que dirige su conocimiento. Estas incorporaciones podrían consolidarse a nivel institucional para visibilizar de manera transversal los principios de la educación patrimonial en todas las carreras de pedagogía de la UCM en tanto espacios que sensibilicen a todos los estudiantes hacia la temática patrimonial, pues, consideramos que es un asunto que compromete a toda la ciudadanía. Finalmente buscamos elaborar un proyecto orientado a la elaboración de políticas institucionales en la UCM para que, en el largo plazo, se cree una conciencia compartida sobre el valor del patrimonio desde la articulación de un sentir ciudadano responsable y comprometido con su entorno social.

\section{REFERENCIAS BIBLIOGRÁFICAS}

Ayala, E. (2019). Eterno resplandor de la memoria y de los derechos. por una pedagogía consciente y reflexiva para la comprensión del presente y de los tiempos que lo cruzan. En A. Ramis \& M. Peña (Coord), Educar para la ciudadanía: fundamentos, metodologías y desarrollo profesional docente. Saberes Docentes, Centro de Estudios y Desarrollo de Educación Continua para el Magisterio de la Universidad de Chile.

Cabrera, H., Muñoz M., \& Reyes P. (2015). Historia de la plaza de armas de Curicó ¿Qué tipo de conocimiento tienen los estudiantes de educación básica acerca de su historia? [Tesis de pregrado]. Universidad Católica del Maule. http://bibliotecavirtualoducal.uc.cl:8081/handle/123456789/1473551

Castro, I., \& López, P. (2020). Lineamientos sobre Educación Patrimonial en la Formación Inicial Docente: Perspectiva de profesores, egresados y estudiantes de Pedagogía en Lengua Castellana y Comunicación [Tesis de grado]. Universidad Católica del Maule.

Delors, J. (1996). La educación encierra un tesoro. Santillana, UNESCO.

DIBAM (2005). Memoria, cultura y creación. Lineamientos políticos. Dirección de Bibliotecas, Archivos y Museos.

Fontal, O. (2003). La educación patrimonial. Teoría y práctica en el aula, el museo e Internet. Trea.

García, Z. (2009). ¿Cómo acercar los bienes patrimoniales a los ciudadanos? Educación Patrimonial, un campo emergente en la gestión del patrimonio cultural. Pasos, 7(2), 271-280. https://doi.org/10.25145/j.pasos.2009.07.019

Hernández, R., Fernández C., \& Baptista M. (2010). Metodología de la investigación. Mc Graw Hill 
Muñoz, E., López, L. (2021). Educación Patrimonial y su inclusión en la Formación Inicial Docente: Una tarea en desarrollo. Revista Convergencia Educativa, (10-extra), diciembre, 7-23. https://doi.org/10.29035/rce.s10.7

Hernández, H. (2020). Fenomenología de las cosas y los símbolos patrimoniales. Uingnam 6, 73-81. http://doi.org/10.22497/quingnam.06.0603

Ibáñez, A., Fontal, O., \& Cuenca, J. (2015). Actualidad y tendencias en Educación Patrimonial. Educatio Siglo XXI, 33(1), 11-14. http://revistas.um.es/educatio/article/view/222471/174721

Ibarra, M., \& Ramírez, C. (2014). Educación patrimonial en Chile. Una propuesta para el desarrollo de la identidad local. Revista América Patrimonio, 6, 39.47. https://repositorio.uc.cl/xmlui/handle/11534/46202

Ley $\mathrm{N}^{\circ}$ 21.045. Diario Oficial de la República de Chile. Santiago, Chile, 3 de noviembre de 2017. https://www.bcn.cl/leychile/navegar?idNorma=1110097

Ley $\mathrm{N}^{\circ}$ 20.911. Diario Oficial de la República de Chile. Santiago, Chile, 2 de abril de 2016. https://www.bcn.cl/leychile/navegar?idNorma=1088963

López, L., Castro, M., \& López, P. (2021). Educación patrimonial y Formación Inicial Docente (FID). Aportes desde el rescate y conservación del patrimonio escolar. Revista Brasileira de Educação, 26, 1-21. https://doi.org/10.1590/S1413-24782021260030

Ramis, A., \& Peña, M. (2019). Educar para la ciudadanía: fundamentos, metodologías y desarrollo profesional docente. Saberes Docentes, Facultad de Filosofía y Humanidades, Universidad de Chile.

UNESCO Etxea. (2004). La UNESCO y el Patrimonio Mundial. UNESCO. https://www.unescoetxea.org/dokumentuak/UNESCOPatrimonio.pdf

UNESCO (1954). Convención para la Protección de los Bienes Culturales en caso de Conflicto Armado, La Haya. https://unesdoc.unesco.org/ark:/48223/pf0000082464

Universidad Católica del Maule (2016). Proyecto Formativo de la Carrera de Pedagogía en Educación General Básica con Mención en Matemática, Lenguaje y Comunicación, Ciencias Naturales o Ciencias Sociales. Universidad Católica del Maule (2013). Proyecto Formativo de la Carrera de Pedagogía en Lengua Castellana y Comunicación. 
Muñoz, E., López, L. (2021). Educación Patrimonial y su inclusión en la Formación Inicial Docente: Una tarea en desarrollo. Revista Convergencia Educativa, (10-extra), diciembre, 7-23. https://doi.org/10.29035/rce.s10.7

\section{Datos de correspondencia}

Enrique Muñoz Reyes

Doctor en Didáctica de la Historia, la Geografía y las Ciencias

Sociales por la Universidad Autónoma de Barcelona.

Universidad Católica del Maule. Curicó, Chile.

Dirección postal: Carmen 682. Curicó, Chile.

ORCID ID: https://orcid.org/0000-0001-6880-4716

Email: emunoz@ucm.cl 